\title{
Comunicação
}

[Communication]

\section{Efeitos genéticos e não-genéticos sobre o intervalo de parto em fêmeas suínas no Sudeste do Brasil}

[Genetic and environmental effects on the farrowing interval in sows in the southeastern region of Brazil]

\author{
A. Cavalcante Neto ${ }^{1,7}$, J.F. Lui $^{2}$, J.L. R. Sarmento ${ }^{3}$, M.N. Ribeiro ${ }^{4}$, J.M.C. Monteiro ${ }^{5}$, \\ C. Fonseca ${ }^{6}$, H. Tonhati ${ }^{2}$ \\ ${ }^{1}$ Aluno de pós-graduação - FCAV-UNESP - Jaboticabal, SP \\ ${ }^{2}$ Faculdade de Ciências Agrárias e Veterinárias - UNESP - Jaboticabal, SP \\ ${ }^{3}$ Universidade Federal do Piauí - Bom Jesus, PI \\ ${ }^{4}$ Universidade Federal Rural de Pernambuco - Recife, PE \\ ${ }^{5}$ Escola Agrotécnica Federal - Muzambinho, MG \\ ${ }^{6}$ Departamento de Biologia/CESAM - UA - Aveiro, Portugal
}

O intervalo de parto é definido como o tempo transcorrido entre um parto e o seguinte. Pode ser calculado em termos médios de rebanho ou para fêmeas individualmente. Apenas pela análise dos dados médios desse intervalo, podem-se avaliar problemas com a eficiência reprodutiva dos animais de uma determinada propriedade. $\mathrm{O}$ maior ou menor valor do intervalo médio de parto de uma matriz é determinante para o maior ou menor número de leitões nascidos, desmamados e, consequentemente, vendidos.

Fatores ambientais e genéticos podem influenciar as características reprodutivas e produtivas dos suínos (Arouca et al., 2004; Brustolini et al., 2004; Pires et al., 2005; Torres Filho et al., 2005a; Torres Filho et al., 2005b; Pires et al., 2008; Cavalcante Neto et al., 2008a). O conhecimento da influência desses fatores sobre tais características torna-se mais importante quando se consideram as diferentes regiões geográficas do Brasil, com clima diversificado, além de manejos deficientes, instalações inadequadas e animais que nem sempre são de alto valor genético (Amaral et al., 2000; Morés et al., 2000; Pinheiro et al., 2000). O objetivo deste trabalho foi o de avaliar os efeitos genéticos e não-genéticos que afetam o intervalo de parto de fêmeas suínas no Sudeste do Brasil.
Para isso, utilizaram-se dados oriundos de fêmeas Dalland (C-40), pertencentes à Granja São José - Irmãos Canalli (rebanho 1), situada no município de Monte Alto, SP, e à Suinocultura São José (rebanho 2), situada no município de Cabo Verde, MG. Os animais estavam todos em instalações convencionais de alvenaria de tijolos, revestidas de cimento e piso impermeável, com piquetes nas unidades destinadas à reprodução. A ração fornecida era do tipo comercial, atendendo às exigências nutricionais de todas as fases da criação.

Para análise dos dados, agruparam-se as estações de parto dos animais em quatro trimestres: 1 janeiro a março; 2 - abril a junho; 3 - julho a setembro; e 4 - outubro a dezembro. Com o intuito de tornar os dados mais consistentes, foram feitas algumas restrições, considerando-se, para análise, apenas informações de reprodutores com, no mínimo, três filhas. Definiu-se, também, que os grupos contemporâneos deveriam incluir, no mínimo, três animais. Feitas as restrições, restaram 1.013 observações de intervalos de parto de 347 porcas filhas de 10 cachaços e 100 matrizes.

A análise dos efeitos ambientais foi realizada pelo procedimento GLM do SAS/1999, considerando-se a opção RANDOM, que trata o efeito de reprodutor como aleatório, utilizando-se

Recebido em 17 de julho de 2008

Aceito em 5 de dezembro de 2008

E-mail: aderbalcavalcante@gmail.com 
um modelo estatístico que pode ser representado por:

$Y_{i j k l m}=\mu+C_{i}+M_{j}+A_{k}+E_{l}+R_{m}+\beta_{l}\left(I_{i j k l m}-I\right)$

$+\beta_{2}\left(P_{i j k l m}-P\right)+\beta_{3}\left(D_{s i j k l m}-D_{s}\right)+\beta_{4}\left(T_{i j k l m}-T\right)$

$+e_{i j k l m n}$, em que:

$\mathrm{Y}_{\mathrm{ijklm}}$ é o valor observado; $\mu$ é a média associada a todas as observações; $\mathrm{C}_{\mathrm{i}}$ é o efeito aleatório do $\mathrm{i}^{\text {-ésimo }}$ cachaço pai da porca dentro de rebanho; $\mathrm{M}_{\mathrm{j}}$ é o efeito aleatório da i- ${ }^{\text {ésima }}$ matriz mãe da porca dentro de rebanho; $A_{k}$ é o efeito fixo do $\mathrm{k}^{\text {-ésimo }}$ ano de parto $(2000, \ldots .2004) ; E_{1}$ é o efeito fixo do $1^{\text {-ésimo }}$ efeito da estação de parição (1, 2, 3 e 4); $\mathrm{R}_{\mathrm{m}}$ é o efeito fixo do $\mathrm{m}^{\text {-ésimo }}$ rebanho (1 e 2); $\beta_{1}$ é o coeficiente de regressão linear da característica em relação à idade da porca ao parto; $\mathrm{I}_{\mathrm{ijklm}}$ é a idade da porca ao parto; I é a média da idade da porca ao parto; $\beta_{2}$ é o coeficiente de regressão linear da característica em relação ao intervalo desmame-cio; $\mathrm{P}_{\mathrm{ijklm}}$ é o intervalo desmame-cio do animal; P é a média do intervalo desmame-cio; $\beta_{3}$ é o coeficiente de regressão linear da característica em relação à duração da lactação; $\mathrm{D}_{\mathrm{ijk} k \mathrm{~m}}$ é a duração da lactação; $\mathrm{D}$ é a média da duração da lactação; $\beta_{4}$ é o coeficiente de regressão linear da característica em relação ao tamanho total da leitegada ao nascer; $\mathrm{T}_{\mathrm{ijklm}}$ é o tamanho total da leitegada ao nascer; $\mathrm{T}$ é a média do tamanho total da leitegada ao nascer; e $e$ é o erro aleatório associado a cada observação, com $\mu=0$ e variância $=\delta^{2}$.

No modelo para análise dos fatores ambientais, testaram-se os efeitos de todas as possíveis interações de efeitos fixos e, como não apresentaram significância no nível de 5\%, foram excluídos das análises. Do mesmo modo, todas as covariáveis foram testadas na forma linear e quadrática, e quando a segunda forma não apresentava significância $(\mathrm{P}>0,05)$, mantinha-se apenas a linear.

Para a análise genética, utilizou-se apenas o primeiro intervalo de parto, o que resultou em 347 observações. O efeito fixo considerado foi grupo de contemporâneos, formados pelo ano de parto, pela estação de parição e, também, pelo rebanho. Como co-variáveis, utilizaram-se a duração da lactação e o intervalo desmame-cio, ambas na forma linear.

As estimativas dos componentes de variância e do parâmetro genético foram obtidas utilizando- se o aplicativo MTDFREML, descrito por Boldman et al. (1995), o qual emprega a metodologia da máxima verossimilhança restrita livre de derivadas. O critério de convergência utilizado foi a variância dos valores do simplex inferior a $10^{-9}$. Após a convergência, o programa era reiniciado, usando-se as estimativas obtidas anteriormente como valores iniciais. Esse procedimento foi repetido até que a diferença entre as estimativas das duas últimas convergências fosse menor que $10^{-9}$. A análise foi realizada sob modelo animal, o qual pode ser representado como segue:

$$
\mathrm{y}=\mathrm{Xb}+\mathrm{Z}_{1} \mathrm{~d}+\mathcal{E}, \text { em que: }
$$

y é o vetor de observações; $X$ é a matriz de incidência de efeitos fixos; $b$ é o vetor de efeitos fixos; $Z_{1}$ é a matriz de incidência de efeitos genéticos aditivos diretos; d é o vetor de efeitos genéticos aditivos diretos; e $\varepsilon$ é o vetor de efeito residual.

As pressuposições assumidas foram que os vetores d e $\varepsilon$ possuem distribuição normal, com $\mathrm{E}(\mathrm{d})=\mathrm{E}(\varepsilon)=0$ e $\operatorname{Var}(\mathrm{d})=A \sigma_{d}^{2}$ e $\operatorname{Var}(\varepsilon)=$ $I_{N} \sigma_{e}^{2}$, sendo $\sigma_{d}^{2}$ e $\sigma_{e}^{2}$ as variâncias genética aditiva direta e residual respectivamente; A é a matriz de numeradores dos coeficientes de parentesco de Wright entre os animais; e $\mathrm{I}_{\mathrm{N}}$ é uma matriz identidade de ordem $\mathrm{N}$, sendo $\mathrm{N}$ o número de observações.

A média e o desvio-padrão para a variável intervalo de parto foram de 140,9 e 5,7 dias respectivamente, com coeficiente de variação de 4,0\%. Roehe e Kennedy (1995), ao trabalharem com animais da raça Yorkshire e Landrace, no Canadá, obtiveram, para o primeiro intervalo de parto, médias de 164,7 e 167 dias, respectivamente. Esses autores afirmaram que esse intervalo foi menor nos partos subsequentes, obtendo, entre o segundo e o terceiro parto, médias de 158,5 e 158,2 para as mesmas raças anteriores e, entre o terceiro e o quarto, de 157,3 e 156,6 também respectivamente.

Não foram observados efeitos de ano de parto ( $P$ $=0,82)$, de estação de parição $(\mathrm{P}=0,76)$, nem de rebanho $(\mathrm{P}=0,63)$ sobre o intervalo de parto, demonstrando, possivelmente, que, quando as marrãs chegam à maturidade, estão menos susceptíveis a esses efeitos, suportando, até certo ponto, diferenças de manejo, ano e estação. 
A duração da lactação exerceu influência linear $(\mathrm{P}<0,0001)$ sobre o intervalo de parto, sendo responsável por $19,4 \%$ da variação total para essa característica. A regressão do intervalo de parto, em função da duração da lactação, revelou aumento de 0,92 dias $(y=107,58+0,92 X)$ no intervalo de parto para cada dia de lactação. Poder-se-ia pensar que um dia de lactação representasse exatamente um dia a mais no intervalo de parto, mas o fato de a duração da lactação não ter coeficiente de regressão linear um pode ser explicado por haver fêmeas suínas que, ao amamentarem, entram em cio antes do desmame, sendo cobertas antes mesmo do término da lactação, embora isso seja raro e represente poucos dias entre a cobertura antecipada e o desmame final. De acordo com Kemp (1998), a fase lactacional de silêncio sexual ocorre devido ao bloqueio neurohormonal sobre a liberação das gonadotrofinas. Logo após o desmame, com a retirada do estímulo da amamentação, começa a secreção gradual dos fatores liberadores de gonadotrofinas pelo hipotálamo e, consequentemente, o reinício da ciclicidade. Segundo Dollanora et al. (2004), em alguns casos, que dependem principalmente do grau de estímulo pela amamentação, da duração da lactação e do estado nutricional da fêmea, pode haver manifestação de estro durante o período de amamentação.

Do mesmo modo, o intervalo desmame-cio relaciona-se linearmente $(\mathrm{P}<0,0001)$ com $\mathrm{o}$ intervalo de parto, justificando $51,7 \%$ da variação total, o que representa um dia a mais para cada dia no intervalo desmame-cio $(y$ $=107,58+1 X)$.

Esses dois últimos resultados eram esperados, visto que estas são as variáveis diretamente relacionadas à quantidade de dias que interrelacionam dois partos. Sobestiansky et al. (1988) afirmaram que, por meio de manejo eficiente, o criador pode controlar as variáveis que regulam o intervalo de parto, ou seja, a duração da lactação e o intervalo desmame-cio.

Adicionalmente, há relatos, na literatura, que mostram que a duração da lactação e o intervalo desmame-cio são características relacionadas. Kummer et al. (2003), por exemplo, agruparam fêmeas suínas em 6 classes, de acordo com a duração da lactação, formando os seguintes grupos: 11-14 dias, 15-16 dias, 17-18 dias, 19-20 dias, 21-26 dias e 27-36 dias. Esses autores observaram que o grupo que apresentou o maior intervalo desmame-cio foi aquele que teve a maior duração da lactação, o que foi confirmado por Cavalcante Neto et al. (2008b). Todavia Cole et al. (1975) e Aumaitre et al. (1976) afirmaram que a duração da lactação relaciona-se negativamente com o intervalo desmame-cio, e esse efeito está claramente presente em lactações menores que uma ou duas semanas. Koketsu e Dial (1997) não observaram diferenças significativas no intervalo desmame-cio com duração da lactação de oito a 19 dias, mas esse intervalo foi menor $(\mathrm{P}<0,05)$ em fêmeas desmamadas aos 20 dias de lactação, com redução maior a partir dos 26 dias. As fêmeas com duração de lactação inferior a sete dias tiveram intervalo desmame-cio maior do que as demais categorias de duração de lactação $(\mathrm{P}<0,05)$.

A regressão linear do número total de leitões nascidos sobre o intervalo de parto não foi significativa $(\mathrm{P}=0,09)$. Do mesmo modo, a regressão do intervalo de parto, em função da idade da porca ao parto, revelou não haver dependência $(\mathrm{P}=0,08)$ entre essas características. Tais fatos podem ser explicados, pelo menos em parte, pela tendência de as fêmeas terem leitegadas menores nas primeiras parições, ao contrário das fêmeas maduras, que tendem a ter leitegadas maiores. Pode haver, então, equivalência da demanda de nutrientes para o crescimento e a manutenção da gestação nas primíparas, em relação à maior demanda de nutrientes das grandes barrigadas nas adultas. Holanda et al. (2005) afirmam que, de fato, há efeito quadrático da idade da porca em relação ao tamanho da leitegada. Esses autores observaram que as maiores leitegadas ocorreram em fêmeas de 2,84 a 3,84 anos de idade, quinta e sexta ordens de parição, estando, inclusive, dentro dos limites observados por Dierckx et al. (1996) e Pinheiro et al. (2000).

$\mathrm{O}$ pai da porca não contribuiu $(\mathrm{P}=0,52)$ para a variação ocorrida no intervalo de parto, ao passo que a mãe da porca causou variação significativa $(\mathrm{P}=0,02)$. Ressalta-se que a variância aqui relacionada é de ordem fenotípica ou total, assim, mesmo havendo diferenças fenotípicas entre as mães, a maior parte dessa diferença pode ser devido ao ambiente e não à genética. 
A estimativa de herdabilidade para o primeiro intervalo de parto foi de 0,00 , o que mostra que o fenótipo não é um bom indicador do genótipo dos indivíduos. Existem, de fato, relatos de estimativas de herdabilidade para essa característica próximas e também iguais a zero. Entre eles, Tholen et al. (1996), que, ao trabalharem com dois rebanhos de suínos na Austrália, encontraram herdabilidade de 0,0 para um rebanho e de 0,10 para o outro. Pode-se citar, ainda, o estudo de Serenius e Stalder (2004), os quais encontraram estimativas de 0,06 e 0,04 também para o primeiro intervalo de partos para as raças Landrace e Large White respectivamente. $\mathrm{Na}$ literatura nacional, foi encontrado apenas um relato de herdabilidade para essa característica, que pertence ao Irgang (1985). Esse autor, por meio da correlação entre meio-irmãs paternas, encontrou herdabilidade de 0,27 , valor bem superior ao do presente estudo.
A diminuição do intervalo de parto melhoraria o desempenho reprodutivo de fêmeas suínas, aumentando, consequentemente, o número de partos/porca/ano. Porém o valor estimado de herdabilidade indica que essa característica não apresentaria ganho genético como resposta à seleção. Entretanto a duração da lactação e o intervalo desmame-cio foram os que justificaram a maior parte da variação ocorrida no intervalo de parto das matrizes. Espera-se que o primeiro sofra basicamente influência de efeitos do meio, ao passo que o segundo apresente influência genética, como mostrado em trabalhos (Tholen et al., 1996; Cavalcante Neto et al., 2008b). Devese, então, para diminuir o intervalo de parto, tentar reduzir, por meio da seleção, o intervalo desmame-cio.

Palavras-chave: suíno, característica reprodutiva, herdabilidade, produtividade

\begin{abstract}
The effect of environmental and genetic effects on the farrowing interval in Dalland (C-40) sows in the Southeast of Brazil was studied. Data consisting of 1,013 farrowing intervals recorded in two herds were analyzed, using a model that included the sire and the dam as random effects and the year of farrowing, the herd and the farrowing season as fixed effects, plus the covariables sow's age at farrowing, litter size at birth, lactation length and weaning-estrus interval. For the farrowing interval first only, variance components were estimated by REML, with an animal model that included, as fixed effect, a contemporary group and, as random effects, the additive genetic variance and the error. The mean farrowing interval was 140.9+5.7 days, with a 4.0\% coefficient of variation. Variance analysis showed no effect of either year, season of farrowing or herd on the farrowing interval. The sire effect was not important for the farrowing interval, but the dam represented an important source of variation. The total number of piglets born and the sow's age at farrowing had no influence on the farrowing interval. The length of lactation exerted an influence on the farrowing interval, accounting for $19.4 \%$ of the total variation of this trait. Likewise, the linear regression of the weaning-estrus interval in relation to the farrowing interval was highly significant, accounting for $51.7 \%$ of the total variation. The heritability estimate was 0.00, suggesting that no genetic gain can be obtained by selection for a shorter farrowing interval.
\end{abstract}

Keywords: swine, heritability, productivity, reproductive trait

O autor A. Cavalcante Neto é bolsista do Programa AlBan/nº E07D402597BR.

\section{REFERÊNCIAS BIBLIOGRÁFICAS}

AMARAL, A.L.; MORÉS, N.; BARIONI JÚNIOR, W. et al. Fatores de risco associados ao desempenho reprodutivo da fêmea suína. Arq. Bras. Med. Vet. Zootec., v.52, p.479-486, 2000.
AROUCA, C.L.C.; FONTES, D.O; FERREIRA,W.M. et al. Exigências de lisina, com base no conceito de proteína ideal, para suínos machos castrados, de 95 a $122 \mathrm{~kg}$, selecionados para deposição de carne magra. Arq. Bras. Med. Vet. Zootec., v.56, p.773-781, 2004. 
AUMAITRE, A.; DARGON, J.; LEGAULT, C. et al. Influence of farm management and breed type on sow's conception-weaning interval and productivity in France. Livest. Product. Sci., v.3, p.75-83, 1976.

BOLDMAN, K.G.; KRIESE, L.A.; Van VLECK, D.L. et al. A manual for use of $M T D F R E M L$. A set of programs to obtain estimates of variances and covariances [DRAFT] Lincoln: USDA/ARS, 1995. 120p.

BRUSTOLINI, P.C.; SILVA, F.C.O.; DONZELE,J.L. Efeitos de fontes lipídicas e níveis de energia digestível sobre o desempenho produtivo e reprodutivo de marras. Arq. Bras. Med. Vet. Zootec., v.56, p.511-521, 2004.

CAVALCANTE NETO, A.; LUI, J.F.; SARMENTO, J.L.R. et al. Efeitos genéticos e ambientais sobre a idade à primeira concepção de fêmeas suínas. Arq. Bras. Med. Vet. Zootec., v.60, p.499-502, 2008a.

CAVALCANTE NETO, A.; LUI, J.F.; SARMENTO, J.L.R. et al. Fatores ambientais e estimativa de herdabilidade para o intervalo desmame-cio em fêmeas suínas. Rev. Bras. Zootec., v.37, p.1953-1958, 2008b

COLE, D.J.A.; VARLEY, M.A.; HUGHES, P.E. Studies in sow reproduction. 2. The effect of lactation length on the subsequent reproductive performance of the sow. Anim. Prod., v.20, p.401-406, 1975.

DIERCKX, S.M.A.G.; RAMOS, A.A.; NUNES, J.R.V. Estudo de fatores de meio sobre características de leitegada em suínos. 1: Tamanho de leitegada. Vet. Zootec., v.8, p.99106, 1996.

DOLLANORA, D.; BERNARDI, L.M.; WENTZ, I. et al. Intervalo desmame-estro e anestro pós-lactacional em suínos: suinocultura em Ação. Porto Alegre: Palloti, 2004. 80p.

HOLANDA, M.C.R.; BARBOSA, S.B.P.; SAMPAIO, J.B.M et al. Tamanho da leitegada e pesos médios ao nascer e aos 21 dias de idade de leitões da raça Large White. Arq. Bras. Med. Vet. Zootec., v.57, p.539-544, 2005.

IRGANG, R. Estimativas de herdabilidade para características que compõem a produtividade anual de leitões por porca. Concórdia: Embrapa Suínos e Aves, 1985. 4p. (Comunicado Técnico, 81).
KEMP, B. Lactational effects on the endocrinology of reproduction. In: VERSTEGEN, M.W.A.; MOUGHAN, P.J.; SCHRAMA, J.W. (Eds). The lactating sow. Wageningen: Wageningen Press, 1998. Cap.13, p.241-257.

KOKETSU, Y.; DIAL, G.D. Factors influencing the postweaning reproductive performance of sows on commercial farms. Theriogenology, v.47, p.1445-1461, 1997.

KUMMER, R.; POLEZE, E.; BERNARDI, M.L. et al. Caracterização do intervalo desmame-estro em uma granja produtora de suínos. In: CONGRESSO DA ASSOCIAÇÃO BRASILEIRA DE VETERINÁRIOS ESPECIALISTAS EM SUÍNOS, 11., 2003, Goiânia. Anais..., Goiânia: ABRAVES, 2003. p.189-190.

MORÉS, N.; SOBESTANSKY, J.; BARIONI JUNIOR, W. et al. Fatores de risco associados aos problemas dos leitões na fase de creche em rebanhos da região Sul do Brasil. Arq. Bras. Med. Vet. Zootec., v.52, p.191-199, 2000.

PINHEIRO, M.J.P.; GALVÃO, R.J.D.; BARBOSA NETO, F. et al. Características reprodutivas de suínos puros na região semiárida do Rio Grande do Norte. I. Tamanho da leitegada. Caatinga, v.13, p.19-26, 2000.

PIRES, A.V.; LOPES, P.S.; GUIMARÃES, S.E.F. et al. Quantitative trait loci mapping for meat quality traits in swine chromosome 6. Arq. Bras. Med. Vet. Zootec., v.57, p.608-615, 2005.

PIRES, A.V.; LOPES, P.S.; GUIMARÃES, S.E.F. et al. Mapeamento de locos de características quantitativas associados à composição de carcaça, no cromossomo seis de suíno. Arq. Bras. Med. Vet. Zootec., v.60, p.725732, 2008.

ROEHE, B.; KENNEDY, B.W. Estimation of genetic parameters for litter size in Canadian Yorkshire and Landrace swine with each parity of farrowing treated as a different trait. J. Anim. Sci., v.73, p.2959-2970, 1995.

SERENIUS, T.; STALDER, K.J. Genetics of length of productive life and lifetime prolificacy in the Finnish Landrace and Large White pig populations. J. Anim. Sci., v.82, p.3111-17, 2004.

SOBESTIANSKY, J.H.; BARBOSA, H.P.; FIALHO, E.T. et al. Efeito do manejo da água e 
da ração ao desmame sobre o intervalo desmame-cio fértil e o número de leitões nascidos. Concórdia: Embrapa - Suínos e Aves, 1988. 2p. (Comunicado Técnico, 136)

TORRES FILHO, R.A.; TORRES, R.A.; LOPES, P.S. et al. Estimativas de parâmetros genéticos para características reprodutivas de suínos. Arq. Bras. Med. Vet. Zootec., v.57, p.684-689, 2005a.

TORRES FILHO, R.A.; TORRES, R.A.; LOPES, P.S. et al. Estimativas de parâmetros genéticos para características de desempenho de suínos em fase de crescimento e terminação. Arq. Bras. Med. Vet. Zootec., v.57, p.237-244, 2005 b.

THOLEN, E.; BUNTER, K.L.; HERMESCH, S. et al. The genetic foundation of fitness and reproduction traits in Australian pig populations 2. Relationships between weaning to conception interval, farrowing interval, stayability, and other common reproduction and production traits. Aust. J. Agri. Res., v.47, p.1275-1290, 1996. 